\title{
BMJ Open Reference architectures for ambient assisted living: a scoping review protocol
}

\author{
Frederico Bublitz (D) , ${ }^{1,2}$ Naman K Sahota, ${ }^{2}$ Arlene Oetomo, ${ }^{2}$ Laura Fadrique, ${ }^{2}$ \\ Plinio P Morita ${ }^{2}$
}

To cite: Bublitz F, Sahota NK, Oetomo A, et al. Reference architectures for ambient assisted living: a scoping review protocol. BMJ Open 2020;10:e033758. doi:10.1136/ bmjopen-2019-033758

- Prepublication history for this paper is available online To view these files, please visit the journal online (http://dx.doi. org/10.1136/bmjopen-2019033758).

Received 20 August 2019 Revised 19 August 2020 Accepted 25 August 2020
Check for updates

(C) Author(s) (or their employer(s)) 2020. Re-use permitted under CC BY-NC. No commercial re-use. See rights and permissions. Published by BMJ.

${ }^{1}$ Department of Computing, State University of Paraiba, Campina Grande, Brazil ${ }^{2}$ School of Public Health and Health Systems, University of Waterloo, Waterloo, Ontario, Canada

Correspondence to Professor Plinio P Morita; plinio.morita@uwaterloo.ca

\section{ABSTRACT}

Introduction For the first time in human history, the number of older people will be higher than the number of children. The prevalence of chronic diseases, such as hypertension, cardiovascular disease, diabetes and mental disorders in older adults is high. Given that, it is essential to make usage of related technology to provide improved health conditions and reduce the costs for promoting ageing in place, and that is precisely the aim of Ambient Assisted Living technology. Considering that these systems provide significant benefit to a vast number of stakeholders, can be applied to the functional diversity of application domains and have high economic and social impacts, it is essential to create reusable and interoperable platforms and standards that are able to deal with the heterogeneity of applications and domains. In this sense, reference architectures have been proposed and evaluated. A comprehensive scoping review concerning the reference architectures must clarify specific aspects, such as what the main domains are and how the solutions effectively deal with them.

Methods This scoping review will follow the methodology framework defined in 'Scoping studies: advancing the methodology'. In this methodological framework, six stages are proposed for scoping review studies: identifying the research question; identifying relevant studies; study selection; charting the data; collating, summarising and reporting the results; and consultation. The research questions aim to investigate what are the motivations, stakeholders, benefits, domains, approaches, architectural components and governance aspects of the proposed reference architectures and models. The team will focus on the Scopus Document Search, PubMed (MEDLINE), IEEE Xplore Digital Library, ACM Digital Library and Science Direct electronic research databases. The search query is a combination of terms related to Ambient Assisted Living AND Reference Architecture. Ethics and dissemination This is a scoping review study and there is no requirement for ethical approval, as primary data will not be collected. The results from this scoping review will be published in a peer-reviewed journal and reported at scientific meetings. We intend to share the results with the International Standards and Conformity Assessment - SyC AAL from Canada to use the review as a basis for establishing an assessment model of reference architectures.

\section{INTRODUCTION}

The world is facing a completely new situation: for the first time in human history, the number of older people will be higher than

\section{Strengths and limitations of this study}

This protocol uses a comprehensive approach, relating seven research questions, that will enable the identification of the main gaps and opportunities in the area.

Due to the comprehensiveness of this review protocol, it can serve as the basis for future works, with more specific scope, and the proposal of standards.

- The investigation of who are the stakeholders associated with the architectural components will enable us to understand how the needs of the stakeholders are fulfilled.

- The inclusion of aspects of data governance in the protocol will serve as the basis for establishing an assessment model of reference architectures.

- One weakness of this review protocol was the need of excluding the term 'architecture' (alone) due to the extensive amount of non-relevant papers found in the rounds for adjusting the search query.

the number of children. ${ }^{1}$ One of the explanations for this statistic is that the number of older people has grown and the prediction is that this number will reach $15 \%$ of the population by $2045 .^{2}$

The fact that we are living longer can be seen as fascinating, yet intimidating at the same time. Though longer lifespans are a clear demonstration of the advances made in science and technology, many questions arise about this unprecedented situation. Are these questions related to important issues like will the ageing population experience decreased quality of life as they age? or how will an ageing population impact the social and economic aspects of society? ${ }^{3}$

Although these questions remain inadequately answered, some aspects of this demographic transition are already known. For instance, chronic diseases are prevalent (eg, hypertension, cardiovascular disease, diabetes and mental disorders) among the elderly. ${ }^{4}$ The prevalence of these chronic diseases alone is already reason enough to harm the 
economy and healthcare system at both individual-level and societal-level, ${ }^{5}$ which indicates the need to continue to evolve for providing improved quality of life in a sustainable way.

A key factor for mitigating the impacts associated with the ageing population is to provide appropriate conditions for older adults to live at home, actively and independently. It should be noted that supporting these conditions for the ageing population is beneficial to all. For the elderly and their family, supportive living at home will allow for better social engagement and fewer expenses related to caregivers and healthcare in general; for the government, assisted living has the potential to reduce costs associated with providing health services and infrastructure for this older population.

Given this context, it is essential to make use of related technology for providing the best conditions and reducing the costs for promoting the ageing in place, and that is precisely the aim of Ambient Assisted Living (AAL) technology. According to Rodriguez et at "AAL refers to concepts, products and services aiming at enhancing several aspects of people's quality of life, including autonomy/independence, comfort, safety, security and health in all stages of their life".

Due to the relevance of AAL systems to the context of the ageing population, it is possible to find a vast amount of reasonable solutions that can benefit people requiring varied levels of assistance. 'But many good ideas and promising pilot cases fail to scale because the adopted approaches have been excessively techno-centric'. ${ }^{7}$ Additionally, there is a trend of these solutions being focussed entirely on the development of a specific and isolated service, for example, the development of a system to monitor some specific health-related parameter, such as the usage of a (or a set of) sensor for fall detection. ${ }^{8}$

In understanding that these systems are beneficial to a vast number of stakeholders, can be applied to functional diversity of application domains, and provide positive economic and social impacts, it is essential to create reusable and interoperable platforms and standards that can counteract the heterogeneity of applications and domains of AAL systems. This will make development of new AAL systems and platforms, that fits the requirements and constraints of the area, easier and faster.

In this sense, some reference architectures have been proposed and evaluated. A reference architecture is "an architecture that encompasses the knowledge about how to design concrete architectures of systems of a given application domain". ${ }^{9}$ For example, Rodriguez et at present "A Comparative Analysis of Reference Architectures for Healthcare in the Ambient Assisted Living Domain", but at the time, only five reference architectures were evaluated and the newest date was from 2011; further, as the authors mention, there is still a lack of reference architectures for supporting some stakeholders such as caregivers and care organisations; and they did not contemplate issues associated with the security of health records. Moreover, as stated by Calvaresi et al in their work, "the solutions often consist of ad-hoc architectures" indicating that a solution is still missing and suggest that solutions should focus on the interaction among all actors instead of the creation of a single solution.

During a recent search (the search was executed on $2^{\text {nd }}$ July 2019.) using a generic query with the terms related to the matter AAL and architecture ('TITLE-ABS-KEY ( ('AAL' OR 'Ambient Assist*') AND ('Conceptual' OR 'Reference Architecture' OR 'Architecture'))) in Scopus Document Search (https://www.scopus.com), it returned with 609 documents. This is indicative of the need to conduct a comprehensive scoping review concerning reference architectures to clarify specific aspects related to identifying the main domains and how the solutions deal with them, understanding who the main stakeholders are and how the solutions include/engage them and the main architectural components of the solution.

\section{METHODS AND ANALYSIS}

This scoping review will follow the methodology framework defined by Levac et al. ${ }^{10}$ In this methodological framework, six stages are proposed for scoping review studies: (1) identifying the research question; (2) identifying relevant studies; (3) study selection; (4) charting the data; (5) collating, summarising and reporting the results; and (6) consultation (optional).

Following this section, the stages of the methodological framework for conducting a scoping study are presented.

\section{Identifying the research question}

The primary objective of this review is to identify the main reference architectures or models and verify whether it is possible to select or establish a comprehensive reference architecture that addresses the most critical challenges of AAL. Due to the comprehensive aspect of this review, a set of related research questions were featured, as follows:

- Motivations: What are the primary purposes of the models/ architecture presented by the papers? For this question, we aim to understand the main concerns that were motivating the authors to create/propose a reference architecture or model for AAL.

- Stakeholders: Who are the stakeholders, and how do the solutions support them? This question aims to identify those that may benefit from the proposed reference architecture. For instance, it is possible that a specific model/architecture has a well-defined focus on the developers of applications for AAL, while others may focus on the people that can benefit from the applications developed using the reference model/architecture. The challenge here is to define a comprehensive definition of the stakeholders that can use or benefit from a reference architecture for AAL.

- Benefits: How do the systems support and deal with the different levels of assistance required by the users (ie, the beneficiaries)? In AAL systems, the beneficiaries may require different levels of assistance. ${ }^{11}$ For instance, they can be independent or require assistance for 
basic daily activities (ADLs), such as for walking or managing their medication.

- Domains: Is the proposed reference architecture or model able to deal with different application domains? Is it possible to specify which ones? Considering that a reference architecture must be able to deal with different application domains inside the AAL scope, such as Personal Care, Remote Patient Monitoring and ADLs, it is important to verify if the architecture is able to deal with these different domains, or if there is some restriction (in this case, this architecture will not be considered for a qualitative analysis).

- Approach: What is the paradigm adopted for the development of the proposed reference architecture or model? A reference architecture can be created based on different approaches or paradigms; for instance, it can adopt a conceptual approach (eg, ontology), a componentbased approach, or a Software as a Service (SaaS) approach.

- Architectural components: What are the main layers or components of the proposed reference architecture? Even considering that the construction of the model or reference architecture can adopt different approaches, it is worthy of listing and correlating them, so that it is possible to find the most common elements, such as support to different Devices, Communications and Clouds. Moreover, it is essential to identify what (and how) components deal with the requirements of heterogeneity, interoperability and reusability.

- Governance: Is there a support mechanism for data governance? For dealing with personal information, a reference architecture should address the main concepts concerning data governance, providing the necessary support for data availability, data consistency, data integrity and data security.

\section{Identifying relevant studies}

This review will be conducted entirely using electronic databases. The following databases were used: Scopus Document Search; PubMed (MEDLINE) (https:// www.ncbi.nlm.nih.gov/pubmed/); IEEE Xplore Digital Library (https://ieeexplore.ieee.org/Xplore/home. jsp); ACM Digital Library (https://dl.acm.org/); and Science Direct (https://www.sciencedirect.com/). These electronic databases were chosen because they cover a vast amount of areas with relevant works related to the scope of this review, such as technology and health. One important note about the choice of the data sources is that the Springer was initially considered to be included; however, most of the papers returned were not relevant as it was not possible to perform an advanced search. In addition to that, the papers returned by Scopus already contemplate papers from Springer.

The research team for this scoping review has extensive experience in the areas of computer science, information technology, pervasive environments, ambient intelligence, ambient assisted living and health sciences. For this review, the team has already performed some rounds for tuning the search string. The main terms related to the AAL and reference architecture. The terms used during this round were: AAL, 'Ambient Intelligence', 'Assisted Living' (which includes all 'Active Assisted Living' papers), 'Ambient Assist*' (that includes all 'Ambient Assisted Living', 'Ambient Assisted' or 'Ambient Assistance' papers), 'Assisted Environment', 'Assistive Environment' (for these latter two terms, the plural form does not impact the results), 'Reference Model', 'Reference Implementation', 'Reference Architecture', 'Conceptual Model' and 'Conceptual Architecture'.

After five rounds of using different combination involving these previous mentioned, and other related, terms and evaluating the relevance of some of the returned papers. Actually, we get 20 papers as control papers, these control papers were articles that we read during these rounds and pre-classified them as relevant, and that should appear in the search. The final search query, used in the Scopus Document Search database as an example, is TITLE-ABS-KEY ('Ambient Intelligence' OR 'AAL' OR 'Assisted Living' OR 'Ambient Assist*' OR 'Assisted Environment' OR 'Assistive Environment') AND ('Reference Model' OR 'Reference Architecture' OR 'Conceptual Model' OR 'Conceptual Architecture').

\section{Study selection}

The focus of this review is to have a comprehensive understanding on how researchers are dealing with, and providing support for, the vast amount of IoT (Internet of Things) devices and services available for AAL, and whether the proposed reference architectures can deal with the main requirements for AAL, as discussed in the research questions.

The team met to discuss the inclusion and exclusion criteria. From these meetings the team has decided that the main restriction with regard to domain is that the paper must either address a solution for solving an AAL problem and mention the software architecture for the solution, or the paper must present a reference architecture (or model) that can be applied in different AAL application domains. The other restrictions are more generic and take into account criteria like availability, language and the type of publication (ie, primary or secondary study). The following inclusion (INC) and exclusion (EXC) criteria were defined:

- INC.01. - Domain: The paper addresses a solution for solving an AAL problem and mentions that there is a software architecture for that.

- INC.02. - Domain: The paper presents a reference architecture (or model) that enables the solution be applied in various AAL application domains.

- INC.03. - Language: The paper must be written in English.

- INC.04. - Time: There are no time restrictions. This option differs a little bit from traditional scoping reviews, which typically include papers from the last 5 years, but the team decided to include any solution 
despite the date of publication to perceive how the theme has evolved over time.

- INC.05. - Study Design: The paper presents a primary study. The team is looking for primary research papers. The team already performed a screening on secondary studies, and, although there are good reviews, there are still gaps that the team overcame by analysing primary studies.

- INC.06. - Availability: The full paper is available. Although the team has access to a considerable amount of data sets, some search engines (eg, Scopus Document Search) point to a third data source where some of the studies may not be available (or only accessible under purchase).

- EXC.01. - Duplicated: The team is using a set of data sets for searching, which may increase the possibility of the paper appearing more than one time. In this case, the team will eliminate the duplicates.

- EXC.02. - not matching all the inclusion criteria: Any paper that does not match all the inclusion criteria will be excluded from the review. The only exception is regarding the domain inclusion criteria, in this case, the paper must fit at least one of them (if not all).

Given the presented inclusion and exclusion criteria, the team will start the screening and eligibility phases based on the flow diagram from PRISMA (Preferred Reporting Items for Systematic Reviews and MetaAnalyses) statement. ${ }^{12}$ For these phases, each paper will be read by two members of the team, and in case of a conflicting decision another member will read the conflicting paper and discuss it with the other members to solve the conflict. Hence, the team will adhere to the following steps for the review: (i) looking for duplicates, maintaining only one version of them; (ii) performing a fast reading on abstract, introduction and conclusion and exclude those studies that clearly fall on one of the exclusion criteria; and (iii) the team will read the paper and exclude those studies that fall on one of the exclusion criteria, explaining the reasons for exclusion.

Regarding the quality assessment of the publication source and its impact, despite that in a first moment, this information will not be used to exclude papers, it is very important to minimise some bias that publications could bring to the review. In this sense, we are going to base this evaluation with the adoption of the study design hierarchy for Software Engineering. ${ }^{13}$

\section{Charting the data}

This stage consists of mapping the information that will be extracted from the primary studies being analysed. ${ }^{14}$ For this stage, the team is using a data chart form using the record information adapted from Crick $e t$ al work, ${ }^{15}$ plus information related to the scope of this review, as follows:

- Bibliographical information: title of the article, authors(s), country, year, as well as the quality of the publication source and its impact.
- Study information: aims of the study, methodology, outcome measures (if applicable), important results.

- AAL information: stakeholders, benefits for stakeholders, level of assistance supported, application domains supported, possibility to extend for other application domains, paradigm or approach used in the study, architectural layers, architectural components, supported elements of data governance and standards adopted.

- Team considerations: strengths of the study, limitations of the study and conclusion.

For extracting the information, each paper will be read by two members of the team; each member will collect the data independently. After that, the collected data for the two distinct members will be merged, and for the conflicting cases, a third member will be consulted to diminish the conflict.

\section{Collating, summarising and reporting the results}

Following the recommendations provided by Levac et $a l^{10}$ this stage will be broken into three steps, as follows:

- Analysis: for this step, the team will provide both quantitative analyses based on the bibliographical information, AAL information and study information data chart criteria; and qualitative analysis by merging $A A L$ information and the team considerations data. For the quantitative analyses, the focus is on the classification of the studies, as a way to answers questions related to all the research questions of this study. The qualitative analysis will use the data from papers and team considerations to extract information that can give a better overview of subjective concepts and opens up new opportunities of studies, for example, we aim to have a deep understanding of how authors perceive the concept of data governance.

- Reporting: for this step the team intend to present a table of strengths and gaps in the evidence; moreover, the structured results combined with the analysis will serve as basis for the creation of a paper.

- Implications for future research, practice and policy: we aim to discuss these results with the International Standards and Conformity Assessment - SyC AAL from Canada focussing on the creation of a reference architecture for AAL systems.

\section{Patient and public involvement}

Patients and/or public were not involved.

\section{DISCUSSION}

This review will allow for the identification of currently used reference architecture and models for AAL, as well as the main aspects concerning their motivations, stakeholders, benefits, domains, approaches, architectural components and governance aspects.

With that information, it will be possible to identify the main gaps in the analysed studies that can serve as the basis for future works and the proposal of standards. It is also expected that this study opens up an opportunity to 
understand the needs of the stakeholders and the aspects of data governance involved to serve as the basis for establishing an assessment model of reference architectures.

Contributors Conceptualisation, methodology and writing - original draft preparation: FB; Writing - review and editing: NKS, AO, LF and PPM.

Funding The authors have not declared a specific grant for this research from any funding agency in the public, commercial or not-for-profit sectors.

Competing interests None declared.

Patient and public involvement Patients and/or the public were not involved in the design, or conduct, or reporting, or dissemination plans of this research.

Patient consent for publication Not required.

Provenance and peer review Not commissioned; externally peer reviewed.

Open access This is an open access article distributed in accordance with the Creative Commons Attribution Non Commercial (CC BY-NC 4.0) license, which permits others to distribute, remix, adapt, build upon this work non-commercially, and license their derivative works on different terms, provided the original work is properly cited, appropriate credit is given, any changes made indicated, and the use is non-commercial. See: http://creativecommons.org/licenses/by-nc/4.0/.

\section{ORCID iD}

Frederico Bublitz http://orcid.org/0000-0003-1711-5392

\section{REFERENCES}

1 Calvaresi D, Cesarini D, Sernani P, et al. Exploring the ambient assisted living domain: a systematic review. J Ambient Intell Humaniz Comput 2017;8:239-57.

2 United Nations. World population prospects - population division - United Nations, 2019. Available: https://population.un.org/wpp/ DataQuery/ [Accessed 8 Jul 2019].
3 World Health Organization. Global health and aging, 2011. Available: https://www.who.int/ageing/publications/global_health.pdf [Accessed 8 Jul 2019].

4 Kleisiaris CF, Papathanasiou IV, Tsaras K, et al. Factors affecting the health status of elderly people receiving home care. Arch Hell Med 2019;36:237-44 https://www.scopus.com/sourceid/27568?origin= recordpage

5 Ge L, Ong R, Yap CW, et al. Effects of chronic diseases on healthrelated quality of life and self-rated health among three adult age groups. Nurs Health Sci 2018;12585.

6 Rodriguez LMG, Ampatzoglou A, Avgeriou P, et al. A comparative analysis of reference architectures for healthcare in the ambient assisted living domain. In: Traina C, Kane B, eds. 2015 IEEE 28th International Symposium on computer-based medical systems. IEEE, 2015: 270-5.

7 Camarinha-Matos LM, Rosas J, Oliveira Al, et al. A collaborative services ecosystem for ambient assisted living. In: IFIP advances in information and communication technology, 2012: 117-27.

8 Camarinha-Matos LM, Ferrada F, Oliveira Al, et al. Integrated care services in ambient assisted living. In: 2013 IEEE 15th International Conference on e-health networking, applications and services (Healthcom 2013), 2013: 197-201.

9 Nakagawa EY, Oquendo F, Maldonado JC. Reference Architectures. In: Oussalah MO, ed. Software architecture: principles, techniques, and tools. 1st edition. London: Wiley, 2014: 88-82.

10 Levac D, Colquhoun H, O'Brien KK. Scoping studies: advancing the methodology. Implement Sci 2010;5:69.

11 Gambi E, Ricciuti M, Ciattaglia G, et al. A technological approach to support the care process of older in residential facilities, 2019.

12 Moher D, Liberati A, Tetzlaff J, et al. Preferred reporting items for systematic reviews and meta-analyses: the PRISMA statement. BMJ 2009;339:b2535.

13 Kitchenham B. Procedures for performing systematic reviews, 2004.

14 Arksey H, O'Malley L. Scoping studies: towards a methodological framework. Int J Soc Res Methodol 2005;8:19-32.

15 Crick M, Angus DE, Backman C. Exploring the role of regulation and the care of older people with depression living in long-term care? A systematic scoping review protocol. BMJ Open 2018;8:e021985. 